\title{
Hard equality constrained integer knapsacks
}

\author{
Karen Aardal* $\quad$ Arjen K. Lenstra ${ }^{\dagger}$
}

February 17, 2005

\begin{abstract}
We consider the following integer feasibility problem: "Given positive integer numbers $a_{0}, a_{1}, \ldots, a_{n}$, with $\operatorname{gcd}\left(a_{1}, \ldots, a_{n}\right)=1$ and $\boldsymbol{a}=\left(a_{1}, \ldots, a_{n}\right)$, does there exist a vector $\boldsymbol{x} \in \mathbb{Z}_{\geq \mathbf{0}}^{n}$ satisfying $\boldsymbol{a x}=a_{0}$ ?" We prove that if the coefficients $a_{1}, \ldots, a_{n}$ have a certain decomposable structure, then the Frobenius number associated with $a_{1}, \ldots, a_{n}$, i.e., the largest value of $a_{0}$ for which $\boldsymbol{a x}=a_{0}$ does not have a nonnegative integer solution, is close to a known upper bound. In the instances we consider, we take $a_{0}$ to be the Frobenius number. Furthermore, we show that the decomposable structure of $a_{1}, \ldots, a_{n}$ makes the solution of a lattice reformulation of our problem almost trivial, since the number of lattice hyperplanes that intersect the polytope resulting from the reformulation in the direction of the last coordinate is going to be very small. For branch-and-bound such instances are difficult to solve, since they are infeasible and have large values of $a_{0} / a_{i}, 1 \leq i \leq n$. We illustrate our results by some computational examples.
\end{abstract}

AMS 2000 Subject classification: Primary: 90C10. Secondary: 45A05, $11 \mathrm{Y} 50$.

OR/MS subject classification: Programming, Integer, Theory.

Key words: Lattice basis reduction, Branching on hyperplanes, Frobenius number.

${ }^{*}$ Centrum voor Wiskunde en Informatica, P.O. Box 94079, 1090 GB Amsterdam, The Netherlands.

${ }^{\dagger}$ Lucent Technologies, Bell Laboratories, 1 North Gate Road, Mendham, NJ 079453104, USA, and Faculteit Wiskunde en Informatica, Technische Universiteit Eindhoven, Postbus 513, 5600 MB Eindhoven, The Netherlands. 


\section{Introduction}

\subsection{Problem Statement and Summary of Results}

Let $a_{0}, a_{1}, \ldots, a_{n}$ be positive integer numbers with $\boldsymbol{a}=\left(a_{1}, \ldots, a_{n}\right)$, $\operatorname{gcd}\left(a_{1}, \ldots, a_{n}\right)=1$ and $a_{i} \leq a_{0}, 1 \leq i \leq n$, and let

$$
P=\left\{\boldsymbol{x} \in \mathbb{R}^{n}: \boldsymbol{a x}=a_{0}, \boldsymbol{x} \geq \mathbf{0}\right\} .
$$

Consider the following integer programming feasibility problem:

Does $P$ contain an integer vector?

If the components of $\boldsymbol{x}$ may take any integer value, then the problem is easy. There exists a vector $\boldsymbol{x} \in \mathbb{Z}^{n}$ satisfying $\boldsymbol{a x}=a_{0}$ if and only if $a_{0}$ is an integer multiple of $\operatorname{gcd}\left(a_{1}, \ldots, a_{n}\right)$. The non-negativity requirement on $\boldsymbol{x}$ makes the problem NP-complete. Examples of problems related to (2) that are very hard to solve by standard methods such as branch-and-bound, include some feasibility problems reported on by Aardal et al. (2000b), certain portfolio planning problems, Louveaux and Wolsey (2002), and the so-called market share problems originally described by Williams (1978) and later stated in a simplified form by Cornuéjols and Dawande (1999). For computational experiments on the Cornuéjols-Dawande instances see also Aardal et al. (2000a).

In this study we focus on infeasible instances to rule out that a search algorithm terminates quickly because it finds a feasible solution by luck. Infeasible instances with large ratios $a_{0} / a_{i}, 1 \leq i \leq n$, are particularly hard for branch-and-bound. The largest value of $a_{0}$ such that the instance of (2) given by the input $a_{1}, \ldots, a_{n}$ is infeasible is called the Frobenius number of $a_{1}, \ldots, a_{n}$, denoted by $F\left(a_{1}, \ldots, a_{n}\right)$. In this context we address two topics. The first one is to provide a sufficient explanation why certain coefficients $a_{1}, \ldots, a_{n}$ will yield larger Frobenius numbers than other coefficients of comparable sizes. In Theorem 2 we demonstrate that the Frobenius number is close to a known upper bound if it is possible to decompose the $\boldsymbol{a}$-coefficients as $a_{i}=M p_{i}+r_{i}$ with $M, p_{i} \in \mathbb{Z}_{>0}, r_{i} \in \mathbb{Z}$, and with $M$ large relative to $p_{i}$ and $\left|r_{i}\right|$.

This leads to the second topic: we give a sufficient condition under which the lattice reformulation using the projection suggested by Aardal, Hurkens, and Lenstra (2000b) will work significantly better than branch-and-bound on instances of type (2). We show that with $a_{0}, a_{1}, \ldots, a_{n}$ as above, the reformulation by Aardal, Hurkens, and Lenstra is computationally very easy to solve in a way similar to Lenstra's algorithm (H.W. Lenstra, Jr. (1983)), 
since the number of lattice hyperplanes intersecting the projected polytope in the direction of the last coordinate is provably small. This is demonstrated in Section 3.2. In the few existing implementations of integer programming algorithm based on Lenstra's idea, typical behavior is that the number of search nodes is smaller than the number of nodes needed by branch-andbound, but every node is more time consuming than a branch-and-bound node due to the computation of a search direction in which the polytope is thin. Here, however, evaluating a node can be done quickly since a thin search direction comes directly from the reformulation. The reformulation, based on lattice basis reduction, is briefly described in Section 2. We also see that instances with $\boldsymbol{a}$-coefficients that decompose in the more general way: $a_{i}=M p_{i}+N r_{i}$ with $M, N, p_{i} \in \mathbb{Z}_{>0}, r_{i} \in \mathbb{Z}$, and with $\left|\boldsymbol{a}^{T}\right|$ large compared to $|\boldsymbol{p}|$ and $|\boldsymbol{r}|$, will be easy to solve after applying the reformulation. Our results are proved using techniques from algebra and number theory.

To illustrate our observations we report on a small computational study on infeasible instances. For all the instances we use the Frobenius number as the right-hand side coefficient $a_{0}$. About half of the instances have $\boldsymbol{a}$ coefficients that decompose as discussed above and in Section 3, and the others have random coefficients of comparable sizes. All instances have $5 \leq n \leq 10$. The computational results, presented in Section 4, clearly confirm our theoretical observations. The decomposable instances are very hard to solve by branch-and-bound since the Frobenius number is large, whereas they become trivial to solve once reformulated since we have a provably thin search direction. The instances with randomly generated $\boldsymbol{a}$-coefficients have much smaller Frobenius numbers, and can be solved reasonably quickly by branch-and-bound. The number of lattice hyperplanes intersecting the reformulated polytope in this case is approximately the same in all coordinate directions, and larger than in the decomposable case. So, for these instances the coordinate directions are not the obvious search directions.

Before presenting our results we will, in the following subsection, give a short description of some known results on integer programming.

\subsection{Integer Programming and Branching on Hyperplanes}

The polytope $P \subseteq \mathbb{R}^{n}$ as defined by (1) has dimension $n-1$, i.e., it is not full-dimensional. In the full-dimensional case the following is known. Let $S$ be a full-dimensional polytope in $\mathbb{R}^{n}$ given by integer input. The width of $S$ along the nonzero vector $\boldsymbol{d}$ is defined as $W(S, \boldsymbol{d})=\max \left\{\boldsymbol{d}^{T} \boldsymbol{x}: \boldsymbol{x} \in\right.$ $S\}-\min \left\{\boldsymbol{d}^{T} \boldsymbol{x}: \boldsymbol{x} \in S\right\}$. Notice that this is different from the definition of the geometric width of a polytope, see e.g. Grötschel, Lovász, and Schrijver 
(1988), p 6.

Consider the problem: "Does the polytope $S$ contain a vector $\boldsymbol{x}$ in the integer lattice $\mathbb{Z}^{n}$ ?" Khinchine (1948) proved that if $S$ does not contain a lattice point, then there exists a nonzero integer vector $\boldsymbol{d}$ such that $W(S, \boldsymbol{d})$ is bounded from above by a constant depending only on the dimension. H. W. Lenstra, Jr., (1983) developed an algorithm, exploiting this fact, for determining whether a given polytope $S$ contains an integer vector or not. The algorithm either finds an integer vector in $S$, or a lattice hyperplane $H$ such that at most $c(n)$ lattice hyperplanes parallel to $H$ intersect $S$, where $c(n)$ is a constant depending only on the dimension $n$. The intersection of each lattice hyperplane with $S$ gives rise to a problem of dimension at most $n-1$, and each of these lower-dimensional problems is solved recursively to determine whether or not $S$ contains an integer vector. One can illustrate the algorithm by a search tree having at most $n$ levels. The number of nodes created at each level is bounded from above by a constant depending only on the dimension at that level. Hence, the algorithm is polynomial for fixed dimension. A search node is pruned if, in the given direction, no lattice hyperplane is intersecting the polytope corresponding to the search node.

There are few implementations of algorithms using the idea of branching on hyperplanes. Gao and Zhang (2002) have implemented Lenstra's algorithm, and Cook et al. (1993) have implemented a heuristic version of the integer programming algorithm by Lovász and Scarf (1992). The Lovász-Scarf algorithm is similar in structure to Lenstra's algorithm. In both implementations one could observe that the number of search nodes created by the algorithms was much less than the number of nodes of a branch-and-bound tree. To compute a good search direction in each node was, however, more time consuming than computing an LP-relaxation. This raises the question of understanding if there are situations in which good search directions can be determined quickly. This is related to one of the results presented in this paper, as we demonstrate that for a class of very difficult infeasible instances, i.e., the instances that have decomposable $\boldsymbol{a}$-coefficients as outlined above, the projection proposed by Aardal, Hurkens, and Lenstra by itself yields an integer direction in which the projected polytope is provably thin. In our case this direction is the last coordinate direction. So, if we apply a tree search algorithm, such as Lenstra's, to the projected polytope, but branch only in coordinate directions in the order of decreasing variable indices, then the instances become very easy. 


\subsection{Notation}

We conclude this section by introducing some definitions and notation. The Euclidean length of a vector $\boldsymbol{x} \in \mathbb{R}^{n}$ is denoted by $|\boldsymbol{x}|$, the $n \times n$ identity matrix by $\boldsymbol{I}^{(n)}$, the zero $p \times q$ matrix by $\mathbf{0}^{(p \times q)}$, where the dimensions are omitted if they are clear from the context.

A set of the form $L=L\left(\boldsymbol{b}_{1}, \ldots, \boldsymbol{b}_{l}\right)=\left\{\sum_{i=1}^{l} \lambda_{i} \boldsymbol{b}_{i}, \lambda_{i} \in \mathbb{Z}, 1 \leq i \leq l\right\}$, where $\boldsymbol{b}_{1}, \ldots, \boldsymbol{b}_{l}$ are linear independent vectors in $\mathbb{R}^{n}, l \leq n$, is called a lattice. The set of vectors $\left\{\boldsymbol{b}_{1}, \ldots, \boldsymbol{b}_{l}\right\}$ is called a lattice basis. A lattice has two different bases if $l=1$, and infinitely many if $l>1$.

The determinant $d(L)$ of the lattice $L$ is defined as $d(L)=\sqrt{\operatorname{det}\left(\boldsymbol{B}^{T} \boldsymbol{B}\right)}$, where $\boldsymbol{B}$ is a basis for $L$, and where $\boldsymbol{B}^{T}$ denotes the transpose of matrix $\boldsymbol{B}$. If the lattice $L$ is full-dimensional we have $d(L)=|\operatorname{det} \boldsymbol{B}|$. The rank of the lattice $L, \operatorname{rk} L$, is the dimension of the Euclidean vector space spanned by $L$. If $\operatorname{rk} L=0$, then $d(L)$ is defined to be equal to one.

The integer width of a polytope $S \subset \mathbb{R}^{n}$ in the non-zero integer direction $\boldsymbol{d} \in \mathbb{Z}^{n}$ is defined as:

$$
W_{I}(S, \boldsymbol{d})=\left\lfloor\max \left\{\boldsymbol{d}^{T} \boldsymbol{x}: \boldsymbol{x} \in S\right\}\right\rfloor-\left\lceil\min \left\{\boldsymbol{d}^{T} \boldsymbol{x}: \boldsymbol{x} \in S\right\}\right\rceil+1 .
$$

The number of lattice hyperplanes in the direction $\boldsymbol{d}$ that intersect $S$ is equal to $W_{I}(S, \boldsymbol{d})$, so if $W_{I}(S, \boldsymbol{d})=0$, then $S$ does not contain an integer vector.

\section{The Reformulation and the Search Algorithm}

The starting point of the reformulation of (2) suggested by Aardal, Hurkens, and Lenstra (2000b) is the sign relaxation $X_{I}=\left\{\boldsymbol{x} \in \mathbb{Z}^{n}: \boldsymbol{a x}=a_{0}\right\}$ of $X=\left\{\boldsymbol{x} \in \mathbb{Z}_{\geq 0}^{n}: \boldsymbol{a x}=a_{0}\right\}$. The relaxation $X_{I}$ can be rewritten as $X_{I}=$ $\left\{\boldsymbol{x} \in \mathbb{Z}^{n}: \boldsymbol{x}=\boldsymbol{x}_{f}+\boldsymbol{B}_{0} \boldsymbol{y}, \boldsymbol{y} \in \mathbb{Z}^{n-1}\right\}$, where $\boldsymbol{x}_{f}$ is an integer vector satisfying $\boldsymbol{a x}_{f}=a_{0}$, and where $\boldsymbol{B}_{0}$ is a basis for the lattice $L_{0}=\left\{\boldsymbol{x} \in \mathbb{Z}^{n}: \boldsymbol{a x}=0\right\}$. That is, there is an integer vector $\boldsymbol{x}_{f}$ such that any vector $\boldsymbol{x} \in X_{I}$ can be written as the sum of $\boldsymbol{x}_{f}$ and a vector $\boldsymbol{x}_{0} \in L_{0}$. Since $\operatorname{gcd}\left(a_{1}, \ldots, a_{n}\right)=1$ and $a_{0}$ is integer, we know that a vector $\boldsymbol{x}_{f}$ exists. In the paper by Aardal et al. it is shown that $\boldsymbol{x}_{f}$ and $\boldsymbol{B}_{0}$ can conveniently be determined in polynomial time using lattice basis reduction.

Let

$$
Q=\left\{\boldsymbol{y} \in \mathbb{R}^{n-1}: \boldsymbol{B}_{0} \boldsymbol{y} \geq-\boldsymbol{x}_{f}\right\} .
$$

Problem (2) can now be restated as:

Does $Q$ contain an integer vector? 
The polytope $Q$ is a full-dimensional formulation, i.e., the dimension of $Q$ is $n-1$, and as mentioned in the previous section we can apply Lenstra's (Lenstra (1983)) algorithm, or any other integer programming algorithm, to $Q$. Here we will consider a tree search algorithm inspired by Lenstra's algorithm, but using only unit directions in the search.

Let $\boldsymbol{e}_{i}, 0 \leq i \leq n-1$, be the $i$ th unit vector, let $J=\{1,2, \ldots, n-1\}$, (assume $n>1$ ) and recursively define a feasibility search process $\operatorname{Search}(S)$ on a set $S \subseteq J$ as follows:

\section{$\operatorname{Search}(S)$ :}

if $S$ is empty, output the point $\left\{k_{j}\right\}_{j \in J}$, print 'feasible' and quit otherwise:

pick an $i \in S$

compute $l_{i}=\left\lceil\min \left\{\boldsymbol{e}_{i}^{T} \boldsymbol{y}: \boldsymbol{y} \in Q\right.\right.$, and $y_{j}=k_{j}$ for all $\left.\left.j \in J \backslash S\right\}\right\rceil$

compute $u_{i}=\left\lfloor\max \left\{\boldsymbol{e}_{i}^{T} \boldsymbol{y}: \boldsymbol{y} \in Q\right.\right.$, and $y_{j}=k_{j}$ for all $\left.\left.j \in J \backslash S\right\}\right\rfloor$

for all integers $k_{i}$ in the interval $\left[l_{i}, u_{i}\right]$ do $\operatorname{Search}(S \backslash\{i\})$

print 'infeasible' and quit

The feasibility search is then defined as $\operatorname{Search}(J)$. For an example of a search tree, see Figure 1. Notice that the search tree created in this way is similar to the search tree of Lenstra's algorithm in that the number of levels of the tree is no more than the number of variables in the problem instance, and that the number of nodes created at a certain level corresponds to the integer width of the polytope in the chosen search direction.

Here we will investigate a class of instances that are exceptionally hard to solve by branch-and-bound when using the original formulation in $\boldsymbol{x}$ variables, but that become easy to solve when applying the branching scheme described above to the reformulated problem in $\boldsymbol{y}$-variables (3). In our implementation of the algorithm $\operatorname{Search}(S)$, we always choose the index $i$ as the highest index in the set $S$ when we are at the step "pick an index $i \in S$ ", i.e., we branch in the order $n-1, \ldots, 1$. This is done because the width in the unit direction $\boldsymbol{e}_{n-1}$ is small for our class of instances as will be demonstrated in following section. Below we give an example of such an instance.

\section{Example 1 Let}

$$
P=\left\{\boldsymbol{x} \in \mathbb{R}^{3}: 12223 x_{1}+12224 x_{2}+36671 x_{3}=149389505, \boldsymbol{x} \geq \mathbf{0}\right\} .
$$




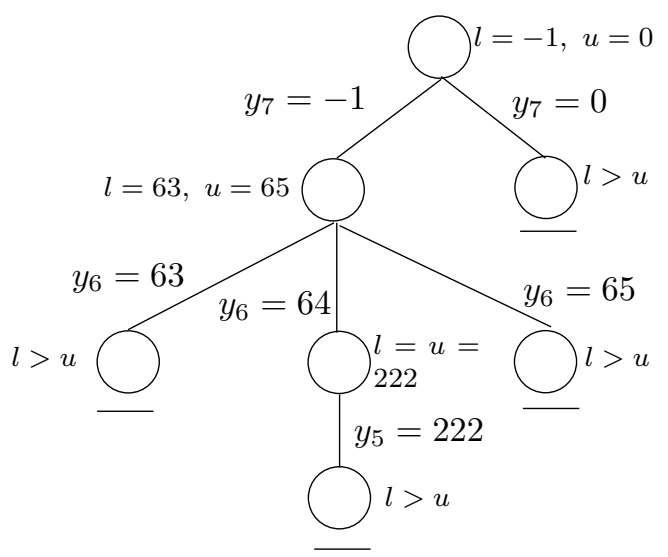

Figure 1: The search tree for instance prob2 (cf. Section 4)

A vector $\boldsymbol{x}_{f}$ and a basis $\boldsymbol{B}_{0}$ for this instance is:

$$
\boldsymbol{x}_{f}=\left(\begin{array}{r}
-4075 \\
4074 \\
4074
\end{array}\right) \quad \boldsymbol{B}_{0}=\left(\begin{array}{rr}
-1 & 14261 \\
-2 & -8149 \\
1 & -2037
\end{array}\right) .
$$

The polytope $Q$ is:

$Q=\left\{\boldsymbol{y} \in \mathbb{R}^{2}:-y_{1}+14261 y_{2} \geq 4075,-2 y_{1}-8149 y_{2} \geq-4074, y_{1}-2037 y_{2} \geq-4074\right\}$.

Moreover, we have $W_{I}\left(Q, \boldsymbol{e}_{1}\right)=4752$ and $W_{I}\left(Q, \boldsymbol{e}_{2}\right)=0$, so if we consider the search direction $\boldsymbol{e}_{2}$ first, we can immediately conclude that $Q \cap \mathbb{Z}^{2}=\emptyset$.

If we solve the formulation in $\boldsymbol{x}$-variables by branch-and-bound with objective function 0 using the default settings of CPLEX 6.5, it takes 1,262,532 search nodes to verify infeasibility.

An instance such as the one given in Example 1 may seem quite artificial. However, some of the instances reported on by Cornuéjols and Dawande (1999), Aardal et al. (2000a,b), and by Louveaux and Wolsey (2000) stem from applications and show a similar behavior. From a practical point of view it is therefore relevant to try to explain this behavior.

\section{The Class of Instances}

In the results presented in this section we will make some, or all, of the assumptions on the input vector $\boldsymbol{a}=\left(a_{1}, \ldots, a_{n}\right)$ that are presented below. 
1. $a_{i} \in \mathbb{Z}, 1 \leq i \leq n$.

2. $1<a_{1}<a_{2}<\cdots<a_{n}$.

3. $\operatorname{gcd}\left(a_{1}, \ldots, a_{n}\right)=1$.

4. $\boldsymbol{a}=M \boldsymbol{p}+\boldsymbol{r}$ with $M, p_{i} \in \mathbb{Z}_{>0}, r_{i} \in \mathbb{Z},\left|r_{i}\right|<M, 1 \leq i \leq n$, and $p_{i}=1$ for at least one $i$.

5. The Hermite normal form of

$$
\boldsymbol{P}=\left(\begin{array}{cccc}
p_{1} & p_{2} & \cdots & p_{n} \\
r_{1} & r_{2} & \cdots & r_{n}
\end{array}\right)
$$

is equal to

$$
\left(\begin{array}{ll}
\boldsymbol{I}^{(2)} & \mathbf{0}^{2 \times(n-2)}
\end{array}\right) .
$$

In Assumption 4 we include $p_{i}=1$ for at least one $i$, as otherwise we could multiply the current $M$ by $\min _{i}\left\{p_{i}\right\}$ to obtain a shorter vector $\boldsymbol{p}$. Assumption 5 implies that $\operatorname{gcd}\left(r_{1}, \ldots, r_{n}\right)=1$, so even if we could find a representation of the vector $\boldsymbol{a}$ as $\boldsymbol{a}=M^{\prime} \boldsymbol{p}^{\prime}+N \boldsymbol{r}^{\prime}$, with a shorter vector $\boldsymbol{r}^{\prime}$ than in the case of the representation $\boldsymbol{a}=M \boldsymbol{p}+\boldsymbol{r}$ satisfying Assumptions 15 , Theorem 2 does not apply to that case. Assumption 5 also implies that the system of equations

$$
\begin{aligned}
& \boldsymbol{p} \boldsymbol{x}=b_{1} \\
& \boldsymbol{r} \boldsymbol{x}=b_{2}
\end{aligned}
$$

has an integer solution $\boldsymbol{x}$ for any integers $b_{1}$ and $b_{2}$. This will be used in the proof of Theorem 2.

\subsection{The Coefficient $a_{0}$}

The polytope $P$ as given in (1) is an $n$-simplex. An instance of problem (2) is particularly hard to solve by branch-and-bound if it is infeasible and if the intersection points of the $n$-simplex with the coordinate axes have large values. Branch-and-bound will then be forced to enumerate many of the possible combinations of $x_{1}, \ldots, x_{n}$ with $0 \leq x_{i} \leq a_{0} / a_{i}$. Since the instance is infeasible we cannot "get lucky" in our search, which may happen if the instance is feasible, and if we by chance have chosen an objective function that takes us to a feasible solution quickly. Example 1 of the previous section illustrates such a hard infeasible instance. Similar, but larger, instances 
are virtually impossible to solve using a state-of-the-art branch-and-bound algorithm such as implemented in CPLEX.

To create infeasible instances with maximum values of $a_{0} / a_{i}$ we choose $a_{0}$ as the Frobenius number $F\left(a_{1}, \ldots, a_{n}\right)$. Computing the Frobenius number for given natural numbers $a_{1}, \ldots, a_{n}$ with $\operatorname{gcd}\left(a_{1}, \ldots, a_{n}\right)=1$ is NPhard (Ramírez Alfonsín (1996)). In Appendix 1 we discuss the algorithm that we used in our computational study. For $n=2$ it is known that $F\left(a_{1}, a_{2}\right)=a_{1} a_{2}-a_{1}-a_{2}$. (In "Mathematics from the Educational Times, with Additional Papers and Solutions", Sylvester published the problem of proving that if $a_{1}$ and $a_{2}$ are relatively prime integers, then there are exactly $\left(a_{1}-1\right)\left(a_{2}-1\right) / 2$ non-negative integers $\alpha$ less than $a_{1} a_{2}-a_{1}-a_{2}$ for which $a_{1} x_{1}+a_{2} x_{2}=\alpha$ does not have a non-negative integer solution. The solution to this problem was provided by Curran Sharp in volume 41 (1884) of the journal. The precise reference is Sylvester and Curran Sharp (1884). See also Schrijver (1986) p. 376.) For $n=3$ the Frobenius number can be computed in polynomial time, see Selmer and Beyer (1978), Rödseth (1978), and Greenberg (1988). Kannan (1992) developed a polynomial time algorithm for computing the Frobenius number for every fixed $n$. His algorithm is based on the relation between the Frobenius number and the covering radius of a certain polytope. Assume $a_{1} \leq a_{2} \leq \cdots \leq a_{n}$. For $n>3$, the value $a_{1} a_{2}-a_{1}-a_{2}$ is an upper bound on $F\left(a_{1}, \ldots, a_{n}\right)$ since it is a valid upper bound for the case $n=2$, and since the Frobenius number can only drop if another term $a_{j} x_{j}$ is added to the Diophantine equation. Other upper bounds on $F\left(a_{1}, \ldots, a_{n}\right)$, and the related case of determining the largest number $a_{0}$ such that $\boldsymbol{a x}=a_{0}$ does not have a solution in positive integers, can be found in the papers by Brauer (1942), Erdős and Graham (1972) and Selmer (1977).

Below we determine a lower bound on $F\left(a_{1}, \ldots, a_{n}\right)$. We express the lower bound as a function of $\boldsymbol{p}, \boldsymbol{r}$ and $M$. The highest order term in $M$ is quadratic in $M$, so for large values of $M$, and relatively small values of $p_{i}$ and $\left|r_{i}\right|$, this term will be dominant. Before presenting the result on the lower bound, we state a lemma.

Lemma 1 Assume $\boldsymbol{a}=M \boldsymbol{p}+\boldsymbol{r}$ satisfies Assumptions 1-5. Let $C$ denote the orthogonal complement of the hyperplane spanned by $\boldsymbol{p}$ and $\boldsymbol{r}$. We denote the lattice $C \cap \mathbb{Z}^{n}$ by $L_{C}$. Then, $L_{C}=L_{0} \cap C$, and $r k L_{C}=n-2$.

Proof: $\quad C=\left\{\boldsymbol{x} \in \mathbb{R}^{n}: \boldsymbol{p} \boldsymbol{x}=0, \boldsymbol{r} \boldsymbol{x}=0\right\}$ is a subspace of $B=\left\{\boldsymbol{x} \in \mathbb{R}^{n}\right.$ : $\boldsymbol{a x}=0\}$.

The fact that $\boldsymbol{x} \in L_{C}$ implies that $\boldsymbol{x} \in C$ and $\boldsymbol{x} \in \mathbb{Z}^{n}$ by the definition of $L_{C}$. Then, in turn, $\boldsymbol{x} \in C$ implies that $\boldsymbol{x} \in B$ since $C$ is a subspace of 
$B$. Hence, $\boldsymbol{x} \in B$ and $\boldsymbol{x} \in \mathbb{Z}^{n}$, which implies that $\boldsymbol{x} \in L_{0}$. Since $\boldsymbol{x} \in C$ and $\boldsymbol{x} \in L_{0}$, we have $\boldsymbol{x} \in L_{0} \cap C$.

If the vector $\boldsymbol{x} \in L_{0} \cap C$, then $\boldsymbol{x} \in \mathbb{Z}^{n}$ as $\boldsymbol{x} \in L_{0}$. From $\boldsymbol{x} \in C$ and $\boldsymbol{x} \in \mathbb{Z}^{n}$ it follows that $\boldsymbol{x} \in L_{C}$.

The rank of $L_{C}$ is equal to $n-2$ due to Assumption 5 .

Theorem 2 Write $a_{i}=M p_{i}+r_{i}, 1 \leq i \leq n$, with $\boldsymbol{a}, \boldsymbol{p}, \boldsymbol{r}$, and $M$ satisfying Assumptions $1-5$ above. Let $\left(r_{j} / p_{j}\right)=\max _{i=1, \ldots, n}\left\{r_{i} / p_{i}\right\}$, and let $\left(r_{k} / p_{k}\right)=\min _{i=1, \ldots, n}\left\{r_{i} / p_{i}\right\}$. We also assume that:

1. $M>2-\left(r_{j} / p_{j}\right)$,

2. $M>\left(r_{j} / p_{j}\right)-2\left(r_{k} / p_{k}\right)$.

Then, we obtain $f(\boldsymbol{p}, \boldsymbol{r}, M) \leq F\left(a_{1}, \ldots, a_{n}\right) \leq g(\boldsymbol{p}, \boldsymbol{r}, M)$, where

$f(\boldsymbol{p}, \boldsymbol{r}, M)=\frac{\left(M^{2} p_{j} p_{k}+M\left(p_{j} r_{k}+p_{k} r_{j}\right)+r_{j} r_{k}\right)\left(1-\frac{2}{M+\left(r_{j} / p_{j}\right)}\right)}{p_{k} r_{j}-p_{j} r_{k}}-\left(M+\frac{r_{j}}{p_{j}}\right)$,

and

$$
g(\boldsymbol{p}, \boldsymbol{r}, M)=M^{2} p_{1} p_{2}+M\left(p_{1} r_{2}+p_{2} r_{1}-p_{1}-p_{2}\right)+r_{1} r_{2}-r_{1}-r_{2} .
$$

Proof: $\quad$ The upper bound $g(\boldsymbol{p}, \boldsymbol{r}, M)$ is derived from the expression

$$
a_{1} a_{2}-a_{1}-a_{2}
$$

In our proof of the lower bound we use the following notation:

$$
\begin{aligned}
B & =\left\{\boldsymbol{x} \in \mathbb{R}^{n}: \boldsymbol{a x}=0\right\} \\
\Delta_{t} & =\left\{\boldsymbol{x} \in \mathbb{R}^{n}: \boldsymbol{a x}=t, \boldsymbol{x} \geq \mathbf{0}\right\} \\
C & =\left\{\boldsymbol{x} \in \mathbb{R}^{n}: \boldsymbol{p} \boldsymbol{x}=0, \boldsymbol{r} \boldsymbol{x}=0\right\} \\
L_{C} & =C \cap \mathbb{Z}^{n} \\
L_{0} & =B \cap \mathbb{Z}^{n} .
\end{aligned}
$$

Notice that the definitions of $B, C$ and $L_{C}$ are as in Lemma 1 , and the definition of $L_{0}$ is as in Section 2. From Lemma 1 we know that $L_{C}=L_{0} \cap C$ and that rk $L_{C}=n-2$.

Before going into the details of the proof we point out that the denominator in the first term of the lower bound $f(\boldsymbol{p}, \boldsymbol{r}, M), p_{k} r_{j}-p_{j} r_{k}$, is not equal to zero. Suppose that the term is equal to zero. This implies that for all $l, 1 \leq l \leq n, l \neq i$ we have $r_{l} / p_{l}=r_{i} / p_{i}=r_{i}=c$, where $i$ is an 


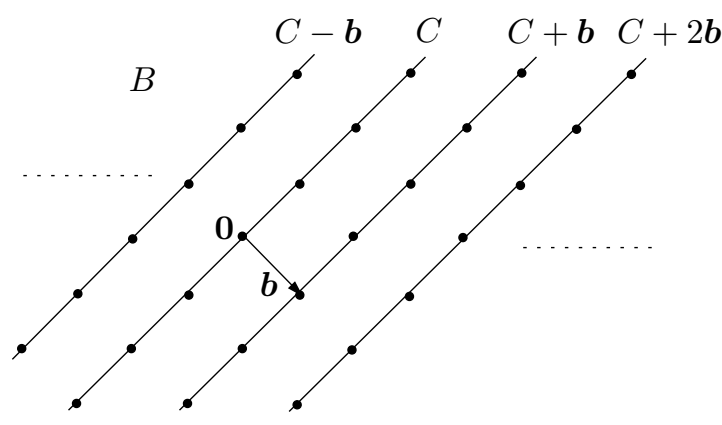

Figure 2: $B$ is the subspace orthogonal to $\boldsymbol{a}=M \boldsymbol{p}+\boldsymbol{r}$, and $C$ is the subspace orthogonal to the plane generated by $\boldsymbol{p}$ and $\boldsymbol{r}$. The lattice $L_{0}$ is contained in parallel hyperplanes generated by $C$ and the lattice basis vector $\boldsymbol{b}$.

index such that $p_{i}=1$. According to Assumption 4, $p_{i}=1$ for at least one $i$. So, $a_{l}=M p_{l}+r_{l}=M p_{l}+p_{l} c=(M+c) p_{l}$. If $(M+c) \neq 1$, then $\operatorname{gcd}\left(a_{1}, \ldots, a_{n}\right) \neq 1$, contradicting Assumption 3. If $(M+c)=1$, then $a_{i}=M p_{i}+c=M+c=1$, contradicting Assumption 2. So, we can conclude that $p_{k} r_{j}-p_{j} r_{k} \neq 0$.

The idea behind the proof is as follows. We define a composition of maps from $\Delta_{t}$ to $\mathbb{R} / \mathbb{Z}$ such that $\boldsymbol{x} \in \mathbb{Z}^{n} \cap \Delta_{t}$ maps to 0 , if such a vector $\boldsymbol{x}$ exists. An integer number $t$ for which 0 is not contained in the image of $\Delta_{t}$ under this map then provides a lower bound on the Frobenius number. We define such a composition of maps by first defining a projection $\pi_{t}$, along a certain vector $\boldsymbol{z}_{t}$, of $\Delta_{t}$ onto $B$, where $\boldsymbol{z}_{t}$ is an integer vector satisfying $\boldsymbol{a} \boldsymbol{z}_{t}=t$, i.e., $\boldsymbol{z}_{t}$ is in the same plane as $\Delta_{t}$. We choose $\boldsymbol{z}_{t}$ integer so that all integer points in the plane $\boldsymbol{a x}=t$ are mapped in $L_{0}$. Then we consider a homomorphism $f: B \rightarrow \mathbb{R} / \mathbb{Z}$ and show that its kernel is $L_{0}+C$. Due to the First Isomorphism Theorem (see e.g. Hungerford (1996), p. 44) we know that $B$ divided out by (ker $f$ ), i.e., $B /\left(L_{0}+C\right.$ ), is isomorphic to $\mathbb{R} / \mathbb{Z}$. Next, we apply the map $\pi_{1}$ to $\Delta_{1}$ and observe that the image of $\pi_{1}\left(\Delta_{1}\right)$ under the isomorphism $B /\left(L_{0}+C\right) \rightarrow \mathbb{R} / \mathbb{Z}$ is an interval $[l, u]$ in $\mathbb{R} / \mathbb{Z}$. Any point in $\Delta_{t} \cap \mathbb{Z}^{n}$ will be in $L_{0}$, and thus under the composition of maps be mapped to zero. Finally, we determine an integer number $t$ such that $[t l, t u]$ does not contain an integer point. The integer $t$ then yields a lower bound on the Frobenius number under the conditions given in the theorem.

We first define a linear map $\pi_{t}: \mathbb{R}^{n} \rightarrow B$ given by $\pi_{t}(\boldsymbol{x})=t \boldsymbol{x}-(\boldsymbol{a x}) \boldsymbol{z}_{t}$, 
where $t \in \mathbb{Z}_{>0}$, and where $\boldsymbol{z}_{t} \in \mathbb{Z}^{n}$ satisfies $\boldsymbol{p} \boldsymbol{z}_{t}=0$ and $\boldsymbol{r} \boldsymbol{z}_{t}=t$, and hence $\boldsymbol{a} \boldsymbol{z}_{t}=t$. Such a vector $\boldsymbol{z}_{t}$ exists due to Assumption 5. Notice that for any $\boldsymbol{x} \in \mathbb{R}^{n}$, the image of $\boldsymbol{x}$ under $\pi_{t}$ is in $B$, i.e., $\boldsymbol{a} \cdot \pi_{t}(\boldsymbol{x})=t(\boldsymbol{a x})-(\boldsymbol{a x})\left(\boldsymbol{a} \boldsymbol{z}_{t}\right)=$ $t(\boldsymbol{a x})-(\boldsymbol{a x}) t=0$. We also observe that if $\boldsymbol{x} \in \mathbb{Z}^{n}$, then $\pi_{t}(\boldsymbol{x})=t \boldsymbol{x}-(\boldsymbol{a x}) \boldsymbol{z}_{t}$ is integer and thus in $L_{0}$, since the number $t$ and the vectors $\boldsymbol{a}$ and $\boldsymbol{z}_{t}$ are all integer.

Next we define the homomorphism $f: B \rightarrow \mathbb{R} / \mathbb{Z}$ given by

$$
\boldsymbol{x} \mapsto(\boldsymbol{p} \boldsymbol{x} \bmod 1) .
$$

Claim 1: The kernel of $f$ is $L_{0}+C$.

First we show that $\left(L_{0}+C\right) \subseteq(\operatorname{ker} f)$. If $\boldsymbol{x} \in L_{0}$ then $\boldsymbol{x} \in \mathbb{Z}^{n}$, which implies $\boldsymbol{p} \boldsymbol{x} \in \mathbb{Z}$, and hence $(\boldsymbol{p} \boldsymbol{x} \bmod 1)=0$. If $\boldsymbol{x} \in C$, then $\boldsymbol{p} \boldsymbol{x}=0$.

Next, we show that $(\operatorname{ker} f) \subseteq\left(L_{0}+C\right)$. Notice that

$$
(\boldsymbol{p} \boldsymbol{x} \bmod 1)=0 \Leftrightarrow \boldsymbol{p} \boldsymbol{x} \text { is integer . }
$$

Consider solutions to the equations

$$
\begin{aligned}
& p \boldsymbol{x}=1 \\
& \boldsymbol{a x}=0
\end{aligned}
$$

All solutions $\boldsymbol{x}$ to system (5)-(6) can be written as $\boldsymbol{x}=\boldsymbol{x}_{p}+\overline{\boldsymbol{x}}$, where $\boldsymbol{x}_{p}$ satisfies (5)-(6), and where $\overline{\boldsymbol{x}}$ is a vector in the nullspace $\boldsymbol{p} \boldsymbol{x}=0, \boldsymbol{a} \boldsymbol{x}=0$, which is exactly the subspace $C$.

Due to Assumption 5 there exists an integer vector $\boldsymbol{x}_{p}$ satisfying

$$
\boldsymbol{p} \boldsymbol{x}_{p}=1, \boldsymbol{r} \boldsymbol{x}_{p}=-M
$$

and hence $\boldsymbol{a} \boldsymbol{x}_{p}=M \boldsymbol{p} \boldsymbol{x}_{p}+\boldsymbol{r} \boldsymbol{x}_{p}=M-M=0$. Therefore, the vector $\boldsymbol{x}_{p}$ satisfies equations (5)-(6), and moreover, $\boldsymbol{x}_{p}$ belongs to the lattice $L_{0}$.

To complete the proof of the claim we notice that the vector $\boldsymbol{y}=t \boldsymbol{x}_{p} \in L_{0}$ satisfies

$$
\begin{aligned}
& \boldsymbol{p y}=t \\
& \boldsymbol{a y}=0,
\end{aligned}
$$

so for any integer $t$, all solutions to system (7)-(8) can be written as the sum of a vector $t \boldsymbol{x}_{p} \in L_{0}$ plus a vector in $C$. 
Due to the First Isomorphism Theorem, the homomorphism $f$ induces an isomorphism $f^{\prime}: B /\left(L_{0}+C\right) \rightarrow \mathbb{R} / \mathbb{Z}$. Below we determine the image of $\Delta_{1}$ under the composition of the mappings $\pi_{1}: \mathbb{R}^{n} \rightarrow B$ and $f: B \rightarrow$ $B /\left(L_{0}+C\right) \rightarrow \mathbb{R} / \mathbb{Z}$.

We use $\boldsymbol{v}_{i}$ to denote vertex $i$ of $\Delta_{1}$. Vertex $\boldsymbol{v}_{i}$, is the vector $\left(0, \ldots, 0,1 / a_{i}\right.$, $0, \ldots, 0)^{T}$, where $1 / a_{i}=1 /\left(p_{i} M+r_{i}\right)$ is the $i$ th component of $\boldsymbol{v}_{i}$. Applying the linear mapping $\pi_{1}$ to $\boldsymbol{v}_{i}$ yields $\pi_{1}\left(\boldsymbol{v}_{i}\right)=\boldsymbol{v}_{i}-\boldsymbol{z}_{1}$. Next, by the homomorphism $\boldsymbol{x} \mapsto(\boldsymbol{p} \boldsymbol{x} \bmod 1), \pi_{1}\left(\boldsymbol{v}_{i}\right)$ becomes

$$
\frac{p_{i}}{M p_{i}+r_{i}}=\frac{1}{M+r_{i} / p_{i}},
$$

because $1 /\left(M+r_{i} / p_{i}\right)<1$ and $\boldsymbol{p} \boldsymbol{z}_{1}=0$. Let $d_{i}$ denote $1 /\left(M+r_{i} / p_{i}\right)$, and recall that $\left(r_{j} / p_{j}\right)=\max _{i=1, \ldots, n}\left\{r_{i} / p_{i}\right\}$, and $\left(r_{k} / p_{k}\right)=\min _{i=1, \ldots, n}\left\{r_{i} / p_{i}\right\}$. Then, since $\Delta_{1}$ is the convex hull of the vertices $\boldsymbol{v}_{i}, 1 \leq i \leq n$, and since $\pi_{1}$ is a linear map and $f$ a homomorphism, the image of $\pi_{1}\left(\Delta_{1}\right)$ under the isomorphism $f^{\prime}$ is an interval $\left[d_{j}, d_{k}\right]$ of length

$$
L=\frac{p_{k} r_{j}-p_{j} r_{k}}{M^{2} p_{j} p_{k}+M\left(p_{j} r_{k}+p_{k} r_{j}\right)+r_{j} r_{k}} .
$$

Now we will demonstrate that there exists an integer $t \geq \frac{1-2 d_{j}}{L}-\frac{1}{d_{j}}$ such that the interval $\left[t d_{j}, t d_{k}\right]$ does not contain an integer point. This implies that $\frac{1-2 d_{j}}{L}-\frac{1}{d_{j}}$ is a lower bound on the Frobenius number. Notice that $1-2 d_{j}>0$ due to Assumption 1 of the theorem.

Let $k=\frac{1-2 d_{j}}{L}$. The interval $\left[I_{1}, I_{2}\right]=\left[k d_{j}, k d_{k}\right]$ has length equal to $1-2 d_{j}$. Let $\ell=\left\lfloor k d_{j}\right\rfloor$. Notice that $\ell \leq I_{1}$. Now define $k^{\prime}=\ell / d_{j}$. The number $k^{\prime}$ satisfies $k-\frac{1}{d_{j}} \leq k^{\prime} \leq k$, and yields an interval $\left[I_{1}^{\prime}, I_{2}^{\prime}\right]=\left[k^{\prime} d_{j}, k^{\prime} d_{k}\right]$ such that $I_{1}^{\prime}$ is integral. The length of $\left[I_{1}^{\prime}, I_{2}^{\prime}\right]$ is at most equal to the length $1-2 d_{j}$ of the interval $\left[I_{1}, I_{2}\right]$. Therefore, $\left[I_{1}^{\prime}, I_{2}^{\prime}+2 d_{j}\right]$ has length at most 1 . Since $I_{1}^{\prime}$ is integral, it follows that $\left(I_{1}^{\prime}, I_{2}^{\prime}+2 d_{j}\right)$ does not contain an integer. Now define $k^{*}=\left\lfloor k^{\prime}\right\rfloor+1$.

Claim 2: The interval $\left[I_{1}^{*}, I_{2}^{*}\right]=\left[k^{*} d_{j}, k^{*} d_{k}\right]$ does not contain an integer point.

First, note that the second assumption of the theorem implies that $d_{k}<2 d_{j}$. Next, if $k^{\prime}$ is integer, then $k^{*} d_{j}=I_{1}^{\prime}+d_{j}$ and $k^{*} d_{k}=k^{\prime} d_{k}+d_{k}=I_{2}^{\prime}+d_{k}$ so that $k^{*} d_{k}<I_{2}^{\prime}+2 d_{j}$. The claim now follows from the fact that $\left(I_{1}^{\prime}, I_{2}^{\prime}+2 d_{j}\right)$ does not contain an integer. 
Finally, if $k^{\prime}$ is not integer, then we have that $k^{\prime} d_{j}<\left\lfloor k^{\prime}\right\rfloor d_{j}+$ $d_{j}=k^{*} d_{j}$ so that $I_{1}^{\prime}=k^{\prime} d_{j}<k^{*} d_{j}$. The remainder of the argument follows the same reasoning as for $k^{\prime}$ integer.

We finally notice that $k^{*}=\left\lfloor k^{\prime}\right\rfloor+1 \geq\left\lfloor k-\frac{1}{d_{j}}\right\rfloor+1 \geq k-\frac{1}{d_{j}}-1+1=k-\frac{1}{d_{j}}$, so we can conclude that $\frac{1-2 d_{j}}{L}-\frac{1}{d_{j}}$ yields a lower bound on the Frobenius number. We obtain

$\frac{1-2 d_{j}}{L}-\frac{1}{d_{j}}=\frac{\left(M^{2} p_{j} p_{k}+M\left(p_{j} r_{k}+p_{k} r_{j}\right)+r_{j} r_{k}\right)\left(1-\frac{2}{M+\left(r_{j} / p_{j}\right)}\right)}{p_{k} r_{j}-p_{j} r_{k}}-\left(M+\frac{r_{j}}{p_{j}}\right)$.

Example 2 The $\boldsymbol{a}$-coefficients in Example 1 decompose as follows. Let $M=12224$.

$$
\begin{aligned}
& a_{1}=M-1 \\
& a_{2}=M+0 \\
& a_{3}=3 M-1 .
\end{aligned}
$$

Theorem 2 yields a lower bound on the Frobenius number equal to 149, 377, 282 and an upper bound equal to the Frobenius number 149, 389, 505. The lower bound is very close to the upper bound.

For all our instances that decompose with vectors $\boldsymbol{p}$ and $\boldsymbol{r}$ that are short compared to $M$, the Frobenius number is large, see the computational study in Section 4. We have computed the lower bound on the Frobenius number for these instances and in all cases it was close to the actual value.

In the following subsection we demonstrate that instances with $\boldsymbol{a}$-coefficients that decompose with large $M$ and relatively short $\boldsymbol{p}$ and $\boldsymbol{r}$ are trivial to solve using the reformulation outlined in Section 2. These are the instances that are extremely hard to solve by branch-and-bound due to the large Frobenius numbers.

\subsection{The Coefficients $a_{1}, \ldots, a_{n}$}

For the further analysis of our class of instances we wish to express the determinant of the lattice $L_{0}$, and that of the sublattice $L_{C}$, in terms of the input. Before presenting our results, we introduce more notation, two definitions, and present some known results. For more details, see for instance Cassels (1997) and Lenstra (2000).

Definition 1 Let $L$ be a lattice in a Euclidean vector space $E$, and let $K$ be a subgroup of $L$. If there exists a subspace $D$ of $E$ such that $K=L \cap D$, then $K$ is called a pure sublattice. 
Definition 2 Let $L$ be a lattice in a Euclidean vector space $E$ with $\operatorname{dim} E=r k L$. Then the dual lattice $L^{\dagger}$ of $L$ is defined as follows:

$$
L^{\dagger}=\left\{\boldsymbol{x} \in E: \boldsymbol{x}^{T} \boldsymbol{y} \in \mathbb{Z} \text { for all } \boldsymbol{y} \in L\right\} .
$$

The dual lattice of a lattice in $E$ is again a lattice in $E$. For a lattice $L$ and its dual $L^{\dagger}$ we have

$$
d(L)=\frac{1}{d\left(L^{\dagger}\right)} .
$$

Suppose that $K$ is a pure sublattice of the lattice $L$. Then the following holds:

$$
d(L)=d(K) \cdot d(L / K) .
$$

Let $L$ be a lattice with dual $L^{\dagger}$, and let $K$ be a pure sublattice of $L$. Then $K^{\perp}=\left\{\boldsymbol{x} \in L^{\dagger}: \boldsymbol{x}^{T} \boldsymbol{y}=0\right.$ for all $\left.\boldsymbol{y} \in K\right\}$, and we can write

$$
K^{\perp}=(L / K)^{\dagger} .
$$

Theorem 3 Assume that $\boldsymbol{a}=\left(a_{1}, \ldots, a_{n}\right)$ satisfies Assumptions 1-3. Then,

$$
d\left(L_{0}\right)=d\left(L\left(\boldsymbol{a}^{T}\right)\right)=\left|\boldsymbol{a}^{T}\right| .
$$

Proof: $\quad$ Take $L$ to be the lattice $\mathbb{Z}^{n}$, and $K$ to be the lattice $L_{0}$. By equation (10) we have $1=d\left(\mathbb{Z}^{n}\right)=d\left(L_{0}\right) \cdot d\left(\mathbb{Z}^{n} / L_{0}\right)$, or equivalently, by equation (9):

$$
d\left(L_{0}\right)=\frac{1}{d\left(\mathbb{Z}^{n} / L_{0}\right)}=d\left(\left(\mathbb{Z}^{n} / L_{0}\right)^{\dagger}\right) .
$$

From (11) we obtain $\left(\mathbb{Z}^{n} / L_{0}\right)^{\dagger}=L_{0}^{\perp}$, and since the dual lattice of $\mathbb{Z}^{n}$ is again $\mathbb{Z}^{n}$ we have $L_{0}^{\perp}=\left\{\boldsymbol{x} \in \mathbb{Z}^{n}: \boldsymbol{x}^{T} \boldsymbol{y}=0\right.$ for all $\left.\boldsymbol{y} \in L_{0}\right\}$. Since $\operatorname{gcd}\left(a_{1}, \ldots, a_{n}\right)=1$ this is exactly the lattice $L\left(\boldsymbol{a}^{T}\right)$ with basis $\boldsymbol{a}^{T}$. So, $L_{0}^{\perp}=d\left(L\left(\boldsymbol{a}^{T}\right)\right)=\sqrt{\boldsymbol{a a}^{T}}=\left|\boldsymbol{a}^{T}\right|$. We have obtained

$$
d\left(L_{0}\right)=d\left(L\left(\boldsymbol{a}^{T}\right)\right) .
$$

This result is also mentioned in Section 3.2 of the survey by Nguyen and Stern (2000).

Remark 1 Notice that $d\left(L_{0}\right)$ can also be computed as $d\left(L_{0}\right)=\sqrt{\operatorname{det}\left(\boldsymbol{B}_{0}^{T} \boldsymbol{B}_{0}\right)}$, where $\boldsymbol{B}_{0}$ is a basis for $L_{0}$. 
We again write $a_{i}=M p_{i}+r_{i}, 1 \leq i \leq n$ with $\boldsymbol{a}, \boldsymbol{p}, \boldsymbol{r}$, and $M$ satisfying Assumptions 1-5. Below we will make use of the matrix $\boldsymbol{P}$ as introduced in expression (4).

Theorem 4 Assume that $\boldsymbol{a}=M \boldsymbol{p}+\boldsymbol{r}$ satisfies Assumptions 1-5. Then,

$$
d\left(L_{C}\right)=d\left(L\left(\boldsymbol{P}^{T}\right)\right)=\sqrt{\operatorname{det}\left(\boldsymbol{P} \boldsymbol{P}^{T}\right)}=\sqrt{|\boldsymbol{p}|^{2} \cdot|\boldsymbol{r}|^{2}-\left(\boldsymbol{p} \boldsymbol{r}^{T}\right)^{2}} .
$$

Proof: This proof follows the same lines as the proof of Theorem 3. Here we choose the lattice $L$ from Definitions 1 and 2 to be the lattice $\mathbb{Z}^{n}$, and the sublattice $K$ to be the lattice $L_{C}$. We have

$$
d\left(L_{C}\right)=d\left(\left(\mathbb{Z}^{n} / L_{C}\right)^{\dagger}\right)=d\left(L_{C}^{\perp}\right),
$$

and since $\left(\mathbb{Z}^{n}\right)^{\dagger}=\mathbb{Z}^{n}$, we obtain $L_{C}^{\perp}=\left\{\boldsymbol{x} \in \mathbb{Z}^{n}: \boldsymbol{x}^{T} \boldsymbol{y}=0\right.$ for all $\left.\boldsymbol{y} \in L_{C}\right\}$. Due to Assumption $5, \boldsymbol{P}^{T}$ forms a basis for $L_{C}^{\perp}$. Hence, we have

$$
d\left(L_{C}\right)=d\left(\left(\mathbb{Z}^{n} / L_{C}\right)^{\dagger}\right)=d\left(L_{C}^{\perp}\right)=d\left(L\left(\boldsymbol{P}^{T}\right)\right) .
$$

The determinant of the lattice $L\left(\boldsymbol{P}^{T}\right)$ is equal to $\sqrt{\operatorname{det}\left(\boldsymbol{P} \boldsymbol{P}^{T}\right)}=$ $\sqrt{|\boldsymbol{p}|^{2} \cdot|\boldsymbol{r}|^{2}-\left(\boldsymbol{p} \boldsymbol{r}^{T}\right)^{2}}$.

Let $\boldsymbol{b}_{0}^{1}, \boldsymbol{b}_{0}^{2}, \ldots, \boldsymbol{b}_{0}^{n-1}$ be a basis for $L_{0}$, and assume without loss of generality that these basis vectors are ordered such that $\boldsymbol{b}_{0}^{1}, \boldsymbol{b}_{0}^{2}, \ldots, \boldsymbol{b}_{0}^{n-2}$ form a basis for the lattice $L_{C}$. Hence, $\boldsymbol{b}_{0}^{n-1}$ does not belong to $L_{C}$. Let $H=\sum_{i=1}^{n-2} \mathbb{R} \boldsymbol{b}_{0}^{i}$ and let $h$ be the distance of $\boldsymbol{b}_{0}^{n-1}$ to $H$. Notice that $h \leq\left|\boldsymbol{b}_{0}^{n-1}\right|$.

Corollary 5 If $\boldsymbol{a}=M \boldsymbol{p}+\boldsymbol{r}$ satisfies Assumptions 1-5, then

$$
\left|\boldsymbol{b}_{0}^{n-1}\right| \geq \frac{\left|\boldsymbol{a}^{T}\right|}{\sqrt{|\boldsymbol{p}|^{2} \cdot|\boldsymbol{r}|^{2}-\left(\boldsymbol{p} \boldsymbol{r}^{T}\right)^{2}}} .
$$

Proof: The following holds:

$$
d\left(L_{0}\right)=d\left(L_{C}\right) \cdot h \leq d\left(L_{C}\right) \cdot\left|\boldsymbol{b}_{0}^{n-1}\right| .
$$

So,

$$
\left|\boldsymbol{b}_{0}^{n-1}\right| \geq \frac{d\left(L_{0}\right)}{d\left(L_{C}\right)}=\frac{d\left(L_{0}\right)}{d\left(L\left(\boldsymbol{P}^{T}\right)\right)}=\frac{\left|\boldsymbol{a}^{T}\right|}{\sqrt{|\boldsymbol{p}|^{2} \cdot|\boldsymbol{r}|^{2}-\left(\boldsymbol{p r}^{T}\right)^{2}}} .
$$

Suppose $\boldsymbol{p}$ and $\boldsymbol{r}$ are short relative to $M$, and hence to $\left|\boldsymbol{a}^{T}\right|$. Lovász' basis reduction algorithm (Lenstra et al. (1982)) yields a basis in which the basis 
vectors are ordered according to increasing length, up to a certain factor. In a basis $\boldsymbol{B}_{0}$ for $L_{0}$, such as we generate it, the first $n-2$ vectors form a basis for the lattice $L_{C}$. These vectors are short, since the basis is reduced and since the determinant of the lattice $L_{C}$ is equal to $\sqrt{|\boldsymbol{p}|^{2} \cdot|\boldsymbol{r}|^{2}-\left(\boldsymbol{p} \boldsymbol{r}^{T}\right)^{2}}$. The length of the last vector of $\boldsymbol{B}_{0}$ will be bounded from below according to Corollary 5.

Example 3 Recall the decomposition of the $\boldsymbol{a}$-coefficients from Examples 1 and 2. Let $M=12224$.

$$
\begin{aligned}
& a_{1}=M-1 \\
& a_{2}=M+0 \\
& a_{3}=3 M-1,
\end{aligned}
$$

so $\boldsymbol{p}=(1,1,3)^{T}$ and $\boldsymbol{r}=(-1,0,-1)^{T}$. The first column of $\boldsymbol{B}_{0},(-1,-2,1)^{T}$, is short. This vector is orthogonal to $\boldsymbol{a}, \boldsymbol{p}$, and $\boldsymbol{r}$. The second, and last, column of $\boldsymbol{B}_{0},(14261,-8149,-2037)^{T}$, is long.

To summarize, if the determinant of the lattice $L_{0}$ is large due to a large value of $M$, then this large value basically has to be contributed by the last vector $\boldsymbol{b}_{0}^{n-1}$ of $\boldsymbol{B}_{0}$. The long vector $\boldsymbol{b}_{0}^{n-1}$ implies a small value of the integral width of $Q$ in the unit direction $\boldsymbol{e}_{n-1}$, so only a few, in fact often zero or one, lattice hyperplanes intersect $Q$ in this direction for the instances we consider. In Example 1 we observed that $W_{I}\left(Q, \boldsymbol{e}_{2}\right)=0$, which immediately gave us a certificate for infeasibility.

It is interesting to notice that if we write

$$
a_{i}=M p_{i}+N r_{i}, \quad \text { for } i=1, \ldots, n,
$$

where $\boldsymbol{a}, \boldsymbol{p}, \boldsymbol{r}, M$, and $N$ satisfy Assumptions 1-3,5, and $p_{i}, M, N \in$ $\mathbb{Z}_{>0}$, and $r_{i} \in \mathbb{Z}, 1 \leq i \leq n$, then Theorem 4 and Corollary 5 still hold.

\section{Computational Results}

To illustrate our results we have solved various instances of type (2). The instances are given in Table 1 . In the first column the instance name is given. Next, in column " $\boldsymbol{a}$ ", the $\boldsymbol{a}$-coefficients are given, and in the last column the Frobenius number can be found. For all the instances we computed the Frobenius number using the algorithm described in Appendix 1.

The instances can be divided into two groups. The first group contains instances cuww1-cuww5 and prob1-prob10, and the second group consists 
Table 1: The instances

\begin{tabular}{|c|c|c|c|c|c|c|c|c|c|c|c|}
\hline Ista & & & & & & $a$ & & & & & $\begin{array}{l}\text { Frobenius } \\
\text { number }\end{array}$ \\
\hline & & & 36674 & 61119 & 85569 & & & & & & \\
\hline & 1213 & $\begin{array}{l}36679 \\
\end{array}$ & $\begin{array}{l}36682 \\
36405\end{array}$ & 48908 & 61139 & $\begin{array}{l}73365 \\
60683\end{array}$ & & & & & \\
\hline v3 & & $\begin{array}{l}24269 \\
13212\end{array}$ & 36405 & & 4c & & 92 & & & & \\
\hline & & & & & & & & 67141 & & & \\
\hline 0 & 250 & 49 & 497 & 621 & 87 & 880 & 113673 & 119 & & & \\
\hline pro & $\begin{array}{l}119 \\
305\end{array}$ & 233 & 306 & 441 & $\begin{array}{r}927 \\
1224\end{array}$ & 1233 & 136 & 140 & & & \\
\hline ro & & 61679 & & & 133404 & 137 & 159 & & & & \\
\hline ro & $\begin{array}{l}48 \\
28 \\
\end{array}$ & $\begin{array}{l}55893 \\
48198\end{array}$ & $\begin{array}{l}62177 \\
80330\end{array}$ & $\begin{array}{l}65 ! \\
91:\end{array}$ & $\begin{array}{r}86 \\
102\end{array}$ & $\begin{array}{r}87 \\
135 \\
\end{array}$ & $\begin{array}{l}102 \\
165\end{array}$ & $\begin{array}{l}109 \\
176\end{array}$ & & & 6 \\
\hline & & 40 & & & & & & & & & \\
\hline Co & & & 34 & & & & & & & & \\
\hline & & & 48 & & & 10 & & & & & \\
\hline ro & & 20 & 29 & 60 & 64 & & & & & & \\
\hline ro & 4 & 707 & 86 & 92 & 978 & 125 & 134 & 141 & & & 106 \\
\hline prob11 & $\begin{array}{l}11615 \\
14770\end{array}$ & $\begin{array}{l}27638 \\
32480\end{array}$ & $\begin{array}{l}32124 \\
75923\end{array}$ & 860 & $\begin{array}{l}53542 \\
85747\end{array}$ & $\begin{array}{r}56230 \\
91772\end{array}$ & $\begin{array}{r}73104 \\
101240\end{array}$ & $\begin{array}{r}73884 \\
115403\end{array}$ & 51 & & \\
\hline PTotor & & 285 & 100 & & & & 95 & & & & \\
\hline & 11 & 142 & & 52 & & & & & & & \\
\hline & & & & & & & & & & & \\
\hline & & 368 & & & & & & & & & \\
\hline & & & & & & & & & & & \\
\hline & & & & & & & & & & & \\
\hline & 20 & & & 496 & & & & & & & \\
\hline & & & & & & & & & & & \\
\hline
\end{tabular}


Table 2: A value of $M$ for instances cuww1-5 yielding short $\boldsymbol{p}$ and $\boldsymbol{r}$

\begin{tabular}{|r|rrrrr|}
\hline & cuww1 & cuww2 & cuww3 & cuww4 & cuww5 \\
\hline$M$ & 12223 & 12228 & 12137 & 13211 & 13429 \\
\hline
\end{tabular}

of instances prob11-prob20. Instances cuww1-cuww5 were generated by Cornuéjols, Urbaniak, Weismantel, and Wolsey (1997), and the remaining instances were generated for this study. For each of the instances cuww1-cuww5 there is a decomposition $a_{i}=M p_{i}+r_{i}$ with short vectors $\boldsymbol{p}$ and $\boldsymbol{r}$. In Table 2 we give values of $M$ that yield short vectors $\boldsymbol{p}$ and $\boldsymbol{r}$ for these instances. Instances prob1-prob10 were generated such that the $\boldsymbol{a}$-coefficients have a decomposition $a_{i}=M p_{i}+N r_{i}$ with short $\boldsymbol{p}$ and $\boldsymbol{r}$ and long $\boldsymbol{a}^{T}$. We randomly generate $M$ from the uniform distribution $U[10000,20000], N$ from $U[1000,2000], p_{i}$ from $U[1,10]$, and $r_{i}$ from $U[-10,10]$.

In contrast, the second group of instances prob11-prob20 were randomly generated such that the $\boldsymbol{a}$-coefficients are of the same size as in prob1-prob10, but they do not necessarily decompose with short vectors $\boldsymbol{p}$ and $\boldsymbol{r}$. We chose the same size of the $\boldsymbol{a}$-coefficients since this yields values of $d\left(L_{0}\right)$ of approximately the same size as for the instances prob1-prob10. For instances prob11-prob20 coefficient $a_{i}$ is randomly generated from $U[10000,150000]$.

We present the computations purely to illustrate how our theoretical results translate into computations. The instances are therefore quite artificial. But, as mentioned in Section 1, other instances stemming from applications show similar, but less extreme, behavior in comparison with the instances reported on here, and our results partly explain this behavior.

The computational results of verifying infeasibility for the instances is reported on in Table 3. For each instance $\boldsymbol{a}$ we used the Frobenius number $F\left(a_{1}, \ldots, a_{n}\right)$ as the right-hand side coefficient $a_{0}$. For each of the instances we computed $d\left(L_{0}\right)$, the length of each of the basis vectors of the basis $\boldsymbol{B}_{0}$, and the number of lattice hyperplanes intersecting $Q$ in the coordinate directions $\boldsymbol{e}_{1}$ and $\boldsymbol{e}_{n-1}$. We then applied the integer branching algorithm described in Section 2 to $Q$. The number of nodes that were generated, and the computing time in seconds are given in the columns "\# Search tree nodes" and "Time". Finally, we attempted to solve the instances, using the original formulation $P$, by standard linear programming based branchand-bound using CPLEX version 6.5.3 . The number of nodes needed by 
branch-and-bound, and the computing time in seconds are reported on in the columns "\# B\&B nodes" and "B\&B time". For the branch-and-bound algorithm we set the node limit to 50 million nodes. If an instance was not solved within this node limit, this is indicated by " $>50 \times 10^{6}$ " in the column "\# B\&B nodes". The time $t$ needed to evaluate the 50 million nodes is then indicated as " $>t$ " in the column "B\&B time". All the computations were carried out on a Sun Ultra 60 Model 2360 workstation with two UltraSPARC-II $359 \mathrm{MHz}$ processors (our implementation is sequential) and $512 \mathrm{MB}$ of memory.

We make the following observations. First, the Frobenius number of the instances cuww1-cuww5 and prob1-prob10 is about two orders of magnitude larger than the Frobenius number of instances prob11-prob20 (see Table $1)$.

Infeasible instances having large values of the intersection points $a_{0} / a_{i}$ between the $n$-simplex $P$ and the coordinate axes are hard for branch-andbound to solve, and the larger these values are, the harder an instance becomes computationally. So, as a class, the first group of instances is harder for branch-and-bound than the second one. In Table 3 we can see that instances cuww1-cuww5 and prob1-prob10 are considerably harder to solve by branch-and-bound than instances prob11-prob20. The presolver of CPLEX claimed infeasibility for instances cuww2 and prob10, but none of the other instances in the first group was solved within the node limit of 50 million nodes. All of the instances prob11-prob20 were solved by branchand-bound within half a million search nodes and one minute of computing time.

We also observe that the shape of the polytope $Q$ is very much influenced by the decomposition of the $\boldsymbol{a}$-coefficients. If the coefficients decompose with short vectors $\boldsymbol{p}$ and $\boldsymbol{r}$ relative to $M$, then the width of the corresponding polytope $Q$ in the unit direction $\boldsymbol{e}_{n-1}$ is very small. This made the instances trivial for our tree search algorithm applied to $Q$. All instances were solved using less than twenty search nodes and a fraction of a second computing time. For instances prob11-prob20 where the $\boldsymbol{a}$-coefficients are generated randomly from a certain interval we observe that the width of $Q$ is of the same magnitude in all unit directions, and in general greater than two. Our tree search algorithm applied to $Q$ therefore needed more nodes and longer computing times than for the first group of instances. For such instances more effort needs to be spent in order to compute good search directions. 
Table 3: Verification of infeasibility

\begin{tabular}{|c|c|c|c|c|c|c|c|c|c|c|c|c|c|c|c|c|}
\hline Instance & $d\left(L_{0}\right)$ & & & & & $\mid b$ & & & & & $W_{I}\left(Q, e_{1}\right)$ & $W_{I}\left(Q, e_{n-1}\right)$ & \# Search & Time & \# B\&B & $B \& B$ \\
\hline \begin{tabular}{|l|} 
cuww1 \\
\end{tabular} & 112700.5 & & & & & & 2.0 & 3.5 & 3.5 & 4823.1 & 1862 & t) & & .001 & \begin{tabular}{|l|} 
nodes \\
$>50 \times 10^{6}$
\end{tabular} & time \\
\hline cuww2 & 119803.3 & & & & & 2.0 & 2.2 & 2.6 & 3.9 & 2922.9 & 1291 & 1 & 3 & .001 & $0^{*}$ & 0.0 \\
\hline cuww3 & 97088.2 & & & & & 2.0 & 2.4 & 2.8 & 4.0 & 2218.0 & 1155 & 2 & 3 & .001 & $>50 \times 10^{6}$ & $>8079.9$ \\
\hline cuww4 & 154638.3 & & & & 1.7 & 2.4 & 2.4 & 4.0 & 3.0 & 2726.8 & 2429 & 1 & 2 & .001 & $50 \times 10^{6}$ & $>7797.5$ \\
\hline cuww5 & 123066.9 & & & 2.0 & 2.2 & 2.0 & 2.2 & 2.6 & 2.8 & 1711.4 & 1279 & 1 & 3 & .001 & $50 \times 10^{6}$ & $>6080.6$ \\
\hline prob1 & 227895.5 & & & 2.0 & 2.2 & 2.6 & 2.6 & 2.8 & 4.7 & 678.4 & 347 & 2 & 7 & .001 & $50 \times 10^{6}$ & $>7912.6$ \\
\hline prob2 & 256849.8 & & & 1.7 & 1.7 & 2.6 & 3.0 & 3.2 & 4.4 & 1016.0 & 274 & 2 & 7 & .001 & $50 \times 10^{6}$ & $>6529.2$ \\
\hline prob3 & 337663.2 & & & 2.2 & 2.4 & 3.0 & 3.0 & 3.3 & 3.6 & 988.4 & 466 & 2 & 11 & .002 & $>50 \times 10^{6}$ & $>6872.1$ \\
\hline prob4 & 226877.3 & & & 2.6 & 2.4 & 2.6 & 2.4 & 3.6 & 3.5 & 1058.4 & 468 & 2 & 8 & .001 & $50 \times 10^{6}$ & $>8432.2$ \\
\hline prob5 & 324461.5 & & & 2.0 & 2.4 & 2.8 & 3.2 & 3.0 & 3.7 & 937.6 & 964 & 2 & 10 & .002 & $50 \times 10^{6}$ & $>8368.4$ \\
\hline prob6 & 191805.0 & 2.0 & 2.0 & 2.2 & 2.2 & 2.4 & 2.2 & 2.8 & 2.6 & 646.6 & 502 & 2 & 8 & .001 & $>50 \times 10^{6}$ & 5550.1 \\
\hline prob7 & 207240.4 & 1.7 & 1.7 & 1.7 & 2.2 & 2.4 & 2.4 & 2.4 & 2.8 & 888.6 & 588 & 2 & 9 & .002 & $50 \times 10^{6}$ & $>5411.5$ \\
\hline prob8 & 288168.2 & 2.2 & 2.2 & 2.2 & 2.6 & 2.6 & 2.2 & 2.4 & 2.4 & 773.4 & 455 & 2 & 7 & .001 & $>50 \times 10^{6}$ & $>5565.4$ \\
\hline prob9 & 235618.6 & 1.7 & 2.8 & 2.8 & 2.6 & 2.4 & 2.4 & 2.4 & 2.8 & 788.6 & 430 & 2 & 18 & .003 & $>50 \times 10^{6}$ & $>6944.7$ \\
\hline prob10 & 363052.5 & 2.0 & 2.2 & 2.2 & 2.4 & 2.2 & 2.6 & 2.4 & 2.4 & 1165.2 & 880 & 2 & 10 & .002 & $0^{*}$ & 0.0 \\
\hline $\begin{array}{l}\text { prob11 } \\
\text { prob12 }\end{array}$ & $\begin{array}{l}225420.4 \\
307211.3\end{array}$ & $\begin{array}{l}3.6 \\
4.4\end{array}$ & $\begin{array}{l}4.0 \\
4.5\end{array}$ & $\begin{array}{l}4.5 \\
4.6\end{array}$ & $\begin{array}{l}4.4 \\
4.4\end{array}$ & $\begin{array}{l}4.6 \\
4.4\end{array}$ & 4.5 & 4.4 & 6.0 & 5.4 & 2 & 4 & 86 & .012 & 445282 & 51.0 \\
\hline prob13 & 266246.9 & 4.6 & 4.2 & 4.6 & 4.0 & 4.8 & 4.6 & 5.3 & 5.1 & 5.8 & 6 & 6 & 41 & & 580565 & $\begin{array}{l}0.0 \\
62.6\end{array}$ \\
\hline prob14 & 286676.3 & 4.4 & 4.1 & $\begin{array}{l}4.0 \\
4.0\end{array}$ & 4.4 & 4.7 & 4.8 & 5.1 & 5.1 & $\begin{array}{l}5.0 \\
5.6\end{array}$ & 9 & 7 & 112 & .0 & 371424 & 43.4 \\
\hline prob15 & 238047.7 & $\begin{array}{l}3.6 \\
3.6\end{array}$ & 4.5 & 4.1 & $\begin{array}{l}3.4+ \\
3.9\end{array}$ & $\begin{array}{l}4.1 \\
3.9\end{array}$ & 5.1 & 4.8 & 5.5 & $\begin{array}{l}6.0 \\
6.0\end{array}$ & 3 & 3 & 66 & .0 & $\begin{array}{l}426692 \\
4262\end{array}$ & 49.4 \\
\hline prob16 & 297717.2 & 4.0 & 3.7 & $\begin{array}{l}7.1 \\
3.7\end{array}$ & 4.2 & 4.2 & 4.2 & 4.6 & 4.7 & 9.7 & $\begin{array}{l}5 \\
3\end{array}$ & 2 & 67 & $.0 \xi$ & 549483 & 61.4 \\
\hline prob17 & 294591.6 & 4.6 & 4.4 & 4.2 & 4.6 & 4.6 & 5.1 & 4.0 & 4.2 & 5.7 & 0 & 4 & 126 & .15 & 218374 & 24.1 \\
\hline prob18 & 300087.6 & 3.5 & $\begin{array}{l}4.4 \\
4.6\end{array}$ & 4.6 & $\begin{array}{l}.00 \\
4.5\end{array}$ & 5.1 & $\begin{array}{l}0.1 \\
5.2\end{array}$ & $\begin{array}{l}4.0 \\
5.5\end{array}$ & 5.0 & 5.8 & 4 & 5 & 90 & .1 & 425727 & 46.9 \\
\hline prob19 & 249577.9 & $\begin{array}{l}.00 \\
3.9\end{array}$ & $\begin{array}{l}4.0 \\
3.7\end{array}$ & $\begin{array}{l}4.0 \\
4.1\end{array}$ & $\begin{array}{l}5.0 \\
5.1\end{array}$ & $\begin{array}{l}0.1 \\
5.2\end{array}$ & $\begin{array}{l}0.2 \\
5.6\end{array}$ & $\begin{array}{l}0.0 \\
4.8\end{array}$ & $\begin{array}{l}5.0 \\
5.5\end{array}$ & $\begin{array}{l}.0 \\
4.6\end{array}$ & $11^{4}$ & $\begin{array}{l}0 \\
6\end{array}$ & 78 & .100 & 255112 & $\begin{array}{l}40.9 \\
27.7\end{array}$ \\
\hline prob20 & 314283.7 & 3.7 & 4.7 & 4.5 & 3.9 & 4.6 & 4.7 & 5.1 & 5.5 & 6.2 & 5 & 3 & 39 & .005 & 423608 & 46.1 \\
\hline
\end{tabular}

*) CPLEX Presolve determines problem is infeasible or unbounded. 


\section{Acknowledgments}

We want to thank Hendrik Lenstra for his valuable suggestions and in particular for the outline of the proof of Theorem 2. We also wish to thank Bram Verweij for providing a framework code, based on his general enumeration library, for our integer branching algorithm.

The research of the first author was partially financed by the project TMR-DONET nr. ERB FMRX-CT98-0202 of the European Community.

\section{References}

Aardal K., R. E. Bixby, C. A. J. Hurkens, A. K. Lenstra, J. W. Smeltink. 2000a. Market split and basis reduction: Towards a solution of the CornuéjolsDawande instances. INFORMS J. Comput. 12 192-202.

Aardal K., C. A. J. Hurkens, A. K. Lenstra. 2000b. Solving a system of diophantine equations with lower and upper bounds on the variables. Math. Oper. Res. 25 427-442.

Brauer A. 1942. On a problem of partitions. Amer. J. Math. 64 299-312.

Brauer A., J. E. Shockley 1962. On a problem of Frobenius. J. Reine Angew. Math. 211 399-408.

Cassels, J. W. S. 1997. An Introduction to the Geometry of Numbers. Second Printing, Corrected. Reprint of the 1971 ed. Springer-Verlag, Berlin, Heidelberg.

Cook, W., T. Rutherford, H. E. Scarf, D. Shallcross. 1993. An implementation of the generalized basis reduction algorithm for integer programming. ORSA J. Comput. 5 206-212.

Cornuéjols G., M. Dawande. 1999. A class of hard small 0-1 programs. INFORMS J. Comput. 11 205-210.

Cornuéjols, G., R. Urbaniak, R. Weismantel, L. A. Wolsey. 1997. Decomposition of integer programs and of generating sets. R. E. Burkard, G. J. Woeginger, eds., Algorithms - ESA '97. Lecture Notes in Computer Science 1284, Springer-Verlag, Berlin, Heidelberg, Germany, 92-103.

Erdős P., R. L. Graham. 1972. On a linear diophantine problem of Frobenius. Acta Arithm. 21 399-408. 
Gao L., Y. Zhang. 2002. Computational experience with Lenstra's algorithm. Technical Report TR02-12, Department of Computational and Applied Mathematics, Rice University, Houston, TX, USA.

Greenberg H. 1988. Solution to a linear Diophantine equation for nonnegative integers. J. Algorithms 9 343-353.

Grötschel M., L. Lovász, A. Schrijver. 1988. Geometric Algorithms and Combinatorial Optimization, Springer-Verlag, Berlin, Germany.

Hungerford T. W. 1996. Algebra; corrected eighth printing. Springer-Verlag, New York, USA.

Kannan R. 1992. Lattice translates of a polytope and the Frobenius Problem. Combinatorica 12 161-177.

Khinchine A. 1948. A quantitative formulation of Kronecker's theory of approximation (In Russian). Izvestiya Akademii Nauk SSR Seriya Matematika 12 113-122.

Lenstra, A. K., H. W. Lenstra, Jr., L. Lovász. 1982. Factoring polynomials with rational coefficients. Math. Ann. 261 515-534.

Lenstra, H. W., Jr. 1983. Integer programming with a fixed number of variables. Math. Oper. Res. 8 538-548.

Lenstra, H. W., Jr. 2000. Flags and lattice basis reduction. C. Casacuberta, R. M. Miró-Roig, J. Verdera, S. Xambó-Descamps, eds., Proceedings of the third European Congress of Mathematics Volume I, Birkhäuser Verlag, Basel, $37-51$.

Lovász, L., H. E. Scarf. 1992. The generalized basis reduction algorithm. Math. Oper. Res. 17 751-764.

Louveaux Q., L. A. Wolsey. 2002. Combining problem structure with basis reduction to solve a class of hard integer programs. Math. Oper. Res. 27 $470-484$.

Nguyen, P. Q., J. Stern. 2000. Lattice reduction in cryptology. W. Bosma, ed., Algorithmic Number Theory: 4th International Symposium, ANTS-IV Leiden, The Netherlands, July 2-7, 2000, Proceedings. Lecture Notes in Computer Science 1838, Springer-Verlag, Berlin, Heidelberg, 85-112. An updated version can be found at URL:

http://www.di.ens.fr/ pnguyen/pub.html 
Ramírez Alfonsín J. L. 1996. Complexity of the Frobenius problem. Combinatorica 16 143-147.

Rödseth Ö. J. 1978. On a linear diophantine problem of Frobenius. J. Reine Angew. Math. 301 171-178.

Schrijver A. 1986. Theory of Linear and Integer Programming, Wiley, Chichester, UK.

Selmer E. S. 1977. On the linear diophantine problem of Frobenius. J. Reine Angew. Math. 293/294 1-17.

Selmer E. S., Ö. Beyer. 1978. On the linear diophantine problem of Frobenius in three variables. J. Reine Angew. Math. 301 161-170.

Sylvester J. J., W.J. Curran Sharp. 1884. [Problem] 7382. Mathematics from the Educational Times, with Additional Papers and Solutions 4121.

Williams, H. P. 1978. Model Building in Mathematical Programming. John Wiley \& Sons Ltd., Chichester.

\section{Appendix 1: Computing the Frobenius Number}

Since the main aim of this paper is not to compute the Frobenius number we use the Frobenius number to create infeasible instances - our approach is quite simple and based on a theorem by Brauer and Shockley (1962). Assume that $a_{i}$ is integer for $1 \leq i \leq n$, that $0<a_{1} \leq a_{2} \leq \cdots \leq a_{n}$, and that $\operatorname{gcd}\left(a_{1}, \ldots, a_{n}\right)=1$. Let $r_{l}$ be the smallest positive integer congruent to $\left(l \bmod a_{1}\right)$ that can be expressed as a non-negative integer combination of $a_{2}, \ldots, a_{n}$. Each residue class modulo $a_{1}$ does contain numbers representable as $a_{2} x_{2}+\cdots+a_{n} x_{n}$ with $x_{i} \in \mathbb{Z}_{\geq 0}$ for $1 \leq i \leq n$. Let $r=\max _{l \in\left\{1,2, \ldots, a_{1}-1\right\}} r_{l}$.

Theorem 6 (Brauer and Shockley (1962).)

$$
F\left(a_{1}, \ldots, a_{n}\right)=r-a_{1} .
$$

Proof: $\quad$ Suppose we can express $r-a_{1}$ as

$$
r-a_{1}=a_{1} x_{1}+a_{2} x_{2}+\cdots+a_{n} x_{n} \text { with } x_{i} \in \mathbb{Z}_{\geq 0}, 1 \leq i \leq n .
$$

Then,

$$
r-a_{1}\left(1+x_{1}\right)=a_{2} x_{2}+\cdots+a_{n} x_{n} \text { with } x_{i} \in \mathbb{Z}_{\geq 0}, 1 \leq i \leq n,
$$


which contradicts that $r$ is the smallest number in its residue class.

Next, take any integer number $N>r-a_{1}$ and assume that $N$ is not an integer multiple of $a_{1}$, in which case we are done. Assume that $N=$ $\left(\ell \bmod a_{1}\right)$ with $\ell \in\left\{1, \ldots, a_{1}-1\right\}$, so we can write $N=p a_{1}+\ell$ for some $p \in \mathbb{Z}_{\geq 0}$. We know that $N$ is greater than or equal to the smallest number in its residue class that can be represented as $a_{2} x_{2}+\cdots+a_{n} x_{n}$ with $x_{i} \in \mathbb{Z}_{\geq 0}$ for $1 \leq i \leq n$, i.e., $N \geq r_{\ell}=q a_{1}+\ell$ for some $q \in \mathbb{Z}_{\geq 0}$. The following holds: $N-r_{\ell}=p a_{1}+\ell-q a_{1}-\ell=a_{1}(p-q)$, and since $N-r_{\ell} \geq 0$ we have $(p-q) \geq 0$. So, $N$ can be written as

$$
N=a_{1}(p-q)+r_{\ell}=a_{1}(p-q)+a_{2} x_{2}+\cdots+a_{n} x_{n} .
$$

with $(p-q) \geq 0$ and $x_{i} \in \mathbb{Z}_{\geq 0}$ for $2 \leq i \leq n$.

For each $l=1, \ldots, a_{1}-1$ we compute the value of $r_{l}$ as:

$$
r_{l}=\min \left\{\sum_{i=2}^{n} a_{i} x_{i}: \sum_{i=2}^{n} a_{i} x_{i}=l+a_{1} x_{1}, \boldsymbol{x} \in \mathbb{Z}_{\geq 0}^{n}\right\} .
$$

Since the instances of type (13) that we tackled are hard to solve by branchand-bound we again applied the reformulation described in Section 2 to each subproblem and solved the reformulated subproblems by branch-and-bound. Notice that the reformulation only has to be determined for $l=1$. The basis for $L=\left\{x \in \mathbb{Z}^{n}:-a_{1} x_{1}+\sum_{i=2}^{n} a_{i} x_{i}=0\right\}$ is independent of $l$, and if we have computed $\boldsymbol{x}_{f}$ for $l=1$, then $l \boldsymbol{x}_{f}$ can be used in the subsequent computations of subproblems $l=2, \ldots, a_{1}-1$. Cornuéjols et al. (1997) used a formulation similar to (13) for computing the Frobenius number, but instead of using the reformulation described in Section 2 combined with branch-and-bound, they used test sets after having decomposed the $\boldsymbol{a}$-coefficients.

In Table 4 we give the computational results for the Frobenius number computations. In the two first columns the instance name and number of variables are given. Then, the computing time and the total number of branch-and-bound nodes needed for all $a_{1}-1$ subproblems are given. Since $a_{1}$ can vary quite a lot, we report on the average number of branch-andbound nodes per subproblem in the last column. 
Table 4: Results for the Frobenius number computations

\begin{tabular}{|l|r|r|r|r|}
\hline Instance & \# Vars & Time & $\begin{array}{l}\text { Total \# } \\
\text { B\&B nodes }\end{array}$ & $\begin{array}{l}\text { Ave. \# nodes } \\
\text { per subprob. }\end{array}$ \\
\hline cuww1 & 5 & 50.0 & 11652 & 1.0 \\
cuww2 & 6 & 62.3 & 25739 & 2.1 \\
cuww3 & 6 & 64.6 & 39208 & 3.2 \\
cuww4 & 7 & 76.3 & 28980 & 2.2 \\
cuww5 & 8 & 130.2 & 210987 & 15.7 \\
prob1 & 8 & 891.3 & 3782264 & 150.9 \\
prob2 & 8 & 90.2 & 53910 & 4.5 \\
prob3 & 8 & 396.2 & 571199 & 14.4 \\
prob4 & 8 & 371.1 & 204191 & 4.2 \\
prob5 & 8 & 257.6 & 349320 & 12.2 \\
prob6 & 10 & 9057.3 & 39164012 & 1901.1 \\
prob7 & 10 & 200.7 & 93987 & 5.0 \\
prob8 & 10 & 304.8 & 577948 & 33.9 \\
prob9 & 10 & 162.6 & 91223 & 24.5 \\
prob10 & 10 & 586.8 & 445777 & 9.8 \\
\hline prob11 & 10 & 241.3 & 577134 & 49.7 \\
prob12 & 10 & 515.8 & 1518531 & 102.8 \\
prob13 & 10 & 391.8 & 998415 & 65.8 \\
prob14 & 10 & 476.7 & 1551241 & 848.6 \\
prob15 & 10 & 418.0 & 1178543 & 89.8 \\
prob16 & 10 & 821.7 & 2063690 & 58.8 \\
prob17 & 10 & 385.4 & 1027115 & 73.1 \\
prob18 & 10 & 567.3 & 1494456 & 73.6 \\
prob19 & 10 & 499.0 & 1289971 & 63.8 \\
prob20 & 10 & 799.2 & 2070667 & 63.4 \\
\hline
\end{tabular}

